\title{
Post-cardiotomy venovenous extracorporeal membrane oxygenation without heparinization
}

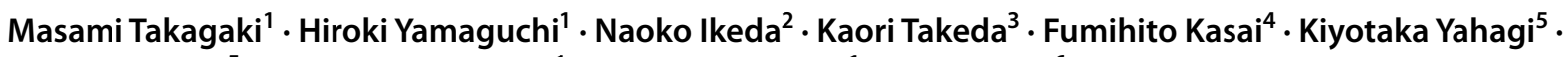 \\ Shunji Kanzaki ${ }^{5} \cdot$ Shinichi Mitsuyama ${ }^{1} \cdot$ Tasuku Kadowaki $^{1} \cdot$ Toru Kotani $^{6}$
}

Received: 25 May 2018 / Accepted: 11 August 2018 / Published online: 17 August 2018

(c) The Author(s) 2018

\begin{abstract}
We present the cases of eight patients (mean age 75 years; EuroSCORE II 17.0 \pm 22.0 ) who underwent post-cardiotomy venovenous extracorporeal membrane oxygenation (ECMO) without heparinization due to serious bleeding. Three liver cirrhosis, two chronic hemodialysis, three redo sternotomy, and two urgent surgery cases were included. Respiratory ECMO Survival Prediction score was $-5.1 \pm 4.2$ (estimated survival rate: approximately 30\%). Mean ECMO duration was 14 days with 9 circuit exchanges. Five patients were weaned from ECMO and three were discharged alive at 90 days (survival 37.5\%). There was a case of pump-head thrombosis requiring urgent circuit exchange. All experienced bleeding complications without clinically apparent pulmonary thromboembolism. Disseminated Intravascular Coagulation scores (Pre $1.3 \pm 0.8$ vs. Post $3.8 \pm 1.7 ; p<0.05)$ significantly increased $(N=6)$. Post-cardiotomy ECMO without heparinization facilitated patient rescue at a reasonable survival rate. However, bleeding complications were still observed. More sophisticated management protocols are warranted.
\end{abstract}

Keywords Extracorporeal membrane oxygenation $\cdot$ Heparinization $\cdot$ Post-cardiotomy $\cdot$ Anticoagulation

\section{Introduction}

We have actively introduced venovenous extracorporeal membrane oxygenation (ECMO) to post-cardiotomy acute respiratory distress syndrome $[1,2]$. However, bleeding is a serious complication that significantly increases the risk of mortality. Bleeding during ECMO support was shown to

Hiroki Yamaguchi

hiroyamaguchiskt@gmail.com

1 Department of Cardiovascular Surgery, Showa University Koto Toyosu Hospital, 5-1-38 Toyosu, Koto-ku, Tokyo 135-8577, Japan

2 Department of Cardiology, Showa University Koto Toyosu Hospital, Tokyo, Japan

3 Department of Nursing, Showa University Koto Toyosu Hospital, Tokyo, Japan

4 Department of Rehabilitation, Showa University Koto Toyosu Hospital, Tokyo, Japan

5 Department of Clinical Engineering, Showa University Koto Toyosu Hospital, Tokyo, Japan

6 Department of Anesthesiology, Showa University Hospital, Tokyo, Japan be related to oxygenator/pump/circuit itself, acquired von Willebrand syndrome, and excessive anticoagulation [3, 4]. Although anticoagulation during ECMO is inevitable to prevent thromboembolic complications, there are no standardized anticoagulation protocols for ECMO till date. In our experience in the cardiac intensive care unit (ICU), the incidence of thromboembolism is very rare compared with that of bleeding, particularly during venovenous ECMO [1, 2]. Furthermore, the cause of major bleeding complications was usually excessive anticoagulation. Herein, we present cases of eight patients who required post-cardiotomy ECMO induction without heparinization due to serious bleeding before and during ECMO. Our management was feasible due to round-the-clock monitoring by at least one physician and clinical engineer to provide urgent circuit exchange.

\section{Cases}

This study was approved by the institutional review board of Showa University Koto Toyosu Hospital. Between April 2014 and August 2017, 10 patients underwent venovenous ECMO without heparinization after undergoing cardiac 
surgery at our institution. Of these, 2 patients were excluded because they required left ventricular assist device implantation for profound left ventricular failure following ECMO induction.

The criteria for induction of venovenous ECMO were as follows: partial pressure of arterial oxygen $\left(\mathrm{PaO}_{2}\right)<80 \mathrm{mmHg}$ and exacerbation of oxygenation or appearance of foamy expectoration under maximum mechanical ventilation [assist control mode; fraction of inspired oxygen $\left(\mathrm{FiO}_{2}\right), 1.0$; positive end-expiratory pressure (PEEP), $10 \mathrm{mmHg}$ ] [1]. Echocardiography helped eliminate structural or functional cardiac abnormality as the cause of hypoxemia. Considering the hemodynamics after major cardiac surgery, PEEP in excess of $10 \mathrm{mmHg}$ was not introduced. ECMO induction was carefully monitored keeping $\mathrm{PaO}_{2}>80 \mathrm{mmHg}$ by increasing PEEP and $\mathrm{FiO}_{2}$. We realize that advanced age is traditionally considered as a relative contraindication for ECMO [5]. However, our patients were preoperatively considered as candidates for cardiac surgery despite their age. We applied ECMO for acute lung damage during the post-operative period regardless of this relative contraindication.

After ECMO induction without heparinization, close bedside monitoring by at least one physician and clinical engineer was ensured $24 \mathrm{~h}$ a day to deal with any potential ECMO-related problem. The pump circuit was examined hourly by registered nurses and/or clinical engineers. Patients in whom hypoxemia improved were evaluated with ECMO without oxygen (room air). If $\mathrm{PaO}_{2}$ was maintained at $>100 \mathrm{mmHg}$ under $\mathrm{FiO}_{2}<0.60$, ECMO was weaned and terminated.

The standard ECMO circuit at our institution consists of a centrifugal pump (MERA Centrifugal Pump NSH-R SENKO MEDICAL INSTRUMENT Mfg. CO., LTD.) and a membrane oxygenator (MERA NHP Excelung NSH-R). In case prolonged support was expected, the X coating (TERUMO's polymer surface coating) system using a CAPIOX SL Centrifugal Pump and a CAPIOX LX Oxygenator (Terumo Corp., Tokyo Japan) was used. Although we did not use heparin, naphamostat mesilate was administered to patients who required continuous hemodialysis. Coumadin was administered in case of atrial fibrillation or mechanical prosthesis.

Continuous variables are expressed as mean \pm standard deviation (median) and categorical variables as frequencies (\%). Paired Student $t$ test was used to assess patients' pre- and post-ECMO data. $p$ values $<0.05$ were considered statistically significant.

The patients (mean age 75 years; all males) were chronologically numbered according to surgery date. Three liver cirrhosis (Child A or B), 2 end-stage renal disease on chronic hemodialysis, 3 redo sternotomy, 2 urgent, and 5 chronic obstructive pulmonary disease cases were included. Consequently, the pre-operative risk scores were very high [EuroSCORE II 17.0 22.0 (11.9); JapanSCORE $21.4 \pm 22.2(14.2)][6,7]$ (Table 1).

Although surgery was performed uneventfully, some patients required prolonged surgical time for hemostasis [surgical time $573 \pm 173(452) \mathrm{min}$ ]. One patient required intra-aortic balloon pump. Cardiopulmonary bypass and aortic clamp times were $284 \pm 114$ (238) and $170 \pm 109$ (200) min, respectively. Acute Physiology and Chronic Health Evaluation (APACHE) II score at ICU admission [8] was $21.8 \pm 9.6(24.5)$, corresponding to an estimated inhospital mortality of $25 \%$ (Table 1 ).

ECMO was induced at mean interval of $15 \pm 11$ (19) days after surgery [intubation time before ECMO: $12 \pm 7$ (12) days]. Reason for no heparinization is described in Table 1. Three patients had surgical bleeding either mediastinal (\#2 and \#8) or retroperitoneal (\#4); another three (\#1, \#3, and \#6) experienced considerable amounts of hemoptysis; and one each had cerebral (\#7) and gastro-intestinal (\#5) bleeding. These pre-ECMO bleeding prompted us to induce ECMO without heparinization. ECMO duration was $14 \pm 16$ (9) days (range 1-47 days) with a total of 114 support days. Five patients were successfully weaned from ECMO. However, 2 of them died during admissions. Three patients were discharged from the hospital and alive at 90 days after ECMO induction (survival rate 37.5\%). (Table 1) All patients received steroids and nitric oxide therapy prior to ECMO induction. ECMO circuit was exchanged 9 times; this included one patient who was weaned and restarted on the same day (patient \#7). The reason for exchange was worsening oxygenation (4), plasma leakage (3), and pumphead thrombosis (1).

Table 1 also summarizes the ratio of $\mathrm{PaO}_{2}$ to $\mathrm{FiO}_{2}(\mathrm{P} / \mathrm{F}$ ratio) and Sequential Organ Failure Assessment (SOFA) score [9] at ECMO induction and after ECMO weaning (or on the day of death of patients who were unable to wean from ECMO) and Murray [10], Respiratory ECMO Survival Prediction (RESP) [11], and APACHE II scores at ECMO induction. No significant difference was observed between the pre- and post-SOFA scores. However, SOFA score tended to decrease in 5 patients who were successfully weaned from ECMO [Pre 14.6 3.9 (14.0) vs. Post $11.2 \pm 5.8(13.0) ; p=0.08]$. Murray and RESP scores at ECMO induction were $3.2 \pm 0.4(3.0)$ and $-5.1 \pm 4.2(-4.0)$, respectively. SOFA and RESP scores at ECMO induction corresponded to an extremely low estimated survival rate $[54 \pm 27 \%(60 \%)$ and $30 \pm 12 \%(32 \%)$, respectively] [9, 11]. APACHE II at ECMO induction was quite high [29.0 \pm 8.7 (28.0)].

All patients experienced episodes of serious bleeding, which is defined as bleeding that requires surgical exploration and/or more than 2 units of red blood cells to maintain hemodynamic control after induction of ECMO. Four patients (\#1, \#5,\#6, and \#7) experienced bleeding from either 


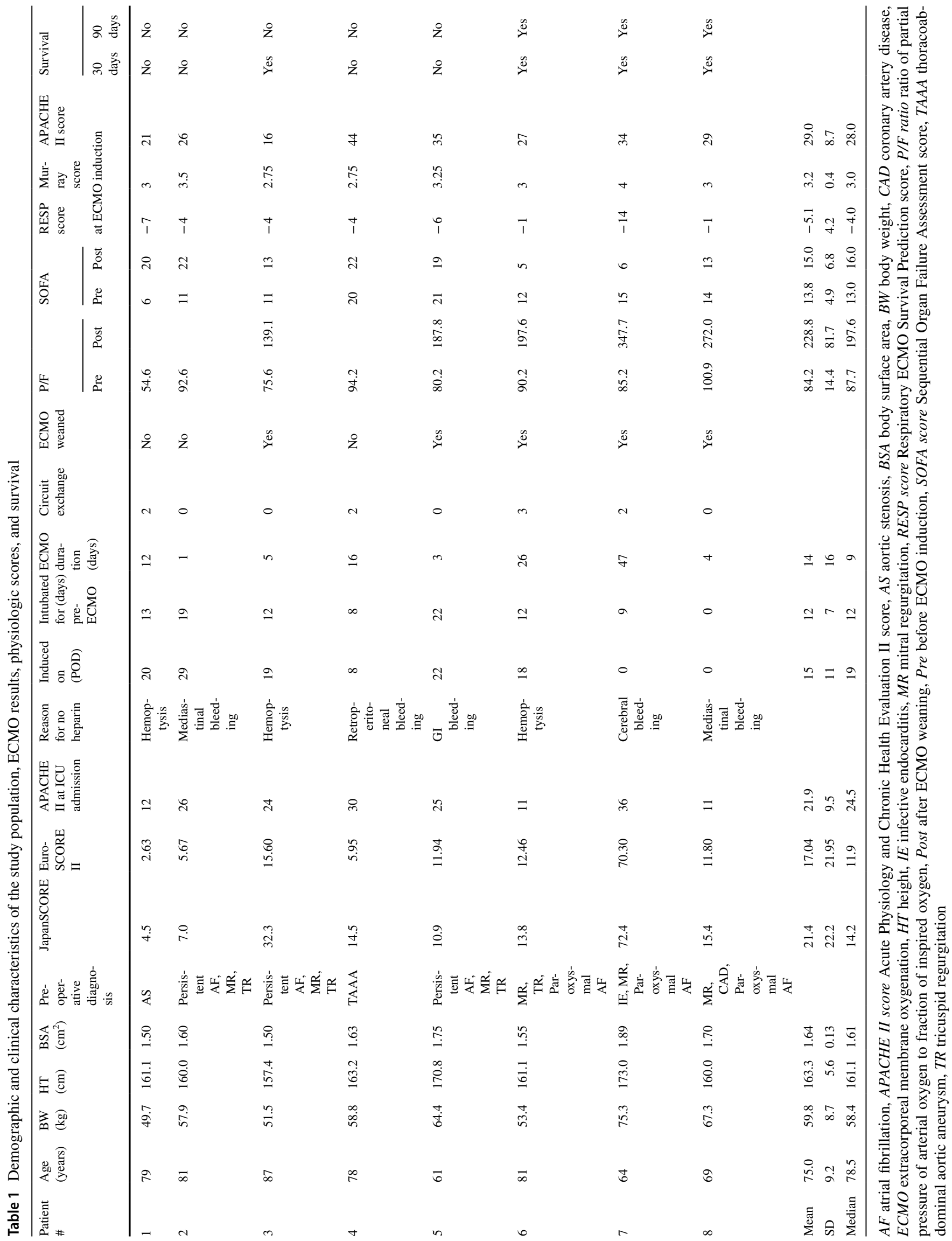


tracheotomy or pulmonary drainage sites; 2 of them required surgical hemostasis. Although bleeding continued even after surgical hemostasis, these complications were spontaneously resolved after weaning from ECMO. Three patients (\#2, \#4, and \#8) experienced progressively worsening surgical bleeding. The pre-ECMO bleeding (Table 1) persisted after ECMO induction in \#3 (hemoptysis) and \#5 (gastro-intestinal bleeding). Clots were observed on the surface of the oxygenator in all cases. There was a case of pump-head thrombosis related to the side branch of the circuit (patient \#7), which required urgent circuit exchange. Post-ECMO observation of the oxygenator in this particular case revealed that the clots were limited to the heat exchanger side (distant from the patient). None of these patients showed clinically apparent episodes of pulmonary thromboembolism.

Pre- and Post-ECMO detailed coagulation data, including antithrombin III, fibrinogen degradation products (FDP), and D-dimer, were available for 6 patients (Patient \# 1, 3, 4, 6,7 , and 8 ) as shown in Table 2. Four of these patients were successfully weaned from ECMO. Although no change in platelet counts was observed due to transfusion, FDP and D-dimer levels significantly increased after a mean duration of 14 days of ECMO support [FDP Pre $8.4 \pm 6.3(11.0) \mu \mathrm{g} /$ $\mathrm{mL}$ vs. Post $56.2 \pm 31.9(65.5) \mu \mathrm{g} / \mathrm{mL} ; p=0.023$; D-dimer Pre $3.2 \pm 2.5(2.7) \mu \mathrm{g} / \mathrm{mL}$ vs. Post $18.4 \pm 11.9$ (16.9) $\mu \mathrm{g} /$ $\mathrm{mL} ; p=0.033]$. Consequently, a significant increase in the Japanese association for acute medicine Disseminated Intravascular Coagulation (DIC) score [12] was observed [Pre $1.3 \pm 0.8$ (1.5) vs. Post $3.8 \pm 1.7$ (4.0); $p=0.022$ ]. Interestingly, daily monitoring of APTT revealed a mild elevation (>35 s) of APTT on 81 days even in the absence of heparin (71\% of total support days).

\section{Discussion}

Although anticoagulation during ECMO is inevitable, [3, 4], recent advances in ECMO technology have allowed the use of minimal anticoagulation with reduced amount of transfusion and without a concomitant increase in mortality. Buscher et al. reported excellent overall survival rates with use of anticoagulation protocol wherein heparin was titrated against a target APTT of 50-70 s with interruption [13]. In a recent study, use of a target APTT of 35-40 s for ECMO management did not cause any major problems [14]. Post-cardiotomy ECMO is frequently complicated by serious bleeding. ECMO therapy with prolonged APTT was shown to be associated with adverse hemorrhagic events such as intracranial hemorrhage [15]. Consequently, we had to induce venovenous ECMO without heparinization to rescue the patients complicated with bleeding.

We took adequate precautions to deal with potential thromboembolism. We ensured round-the-clock monitoring of the patients as well as the ECMO circuits by at least one physician and a clinical engineer; they evaluated the oxygenation capability and pump circuit resistance on an hourly basis. Artificial lung surface thrombosis is quite common, and we daily evaluate its degree, as well. We sometimes decide preemptive pump circuit exchange based on such information. Furthermore, our team has the capability to exchange the circuit urgently (within an hour) $24 \mathrm{~h}$ a day. In this study, pump-head thrombosis was detected on the 22nd day of patient \#7, which was urgently managed by circuit exchange. Indeed, such circumstances enabled us to provide ECMO without heparinization.

Based on pre-operative EuroSCORE II and JapanSCORE, our patients were extremely high-risk patients (expected mortality 20\%). Based on the APACHE II scores at ICU admission, the expected mortality was $25 \%$, which is very similar to that expected from EuroSCORE II and JapanSCORE; this suggests that the surgery went well in the operating room. However, APACHE II score at ECMO induction increased to 29, which was quite high. Murray and RESP scores at ECMO induction showed reasonable indications for ECMO, and based on the RESP and SOFA scores, the expected survival rate was approximately $30 \%$ and $50 \%$, respectively. Considering the severity of our patients based on these scoring systems, our survival rate of $37.5 \%$ seems reasonable.

However, our cases also show that bleeding complications were quite common even without heparinization. Especially, all 3 patients (\#4, 6, and 7) who required prolonged support ( $>2$ weeks) showed lethal bleeding complications, which required a lot of transfusion and/or surgical hemostasis. DIC scores significantly increased after ECMO induction with significant elevation of FDP and D-dimer levels in 6 patients for whom the data were available. Nonetheless, these findings indicate the need to develop more sophisticated anticoagulation protocols for post-cardiotomy venovenous ECMO. Interestingly, APTT was mildly elevated ( $>35 \mathrm{~s}$ ) on $71 \%$ of the total days of support time even without heparin. This may be attributable to the underlying liver cirrhosis, anticoagulation for atrial fibrillation, mechanical prosthesis, and continuous hemodialysis. Identification of the bleeding risks (related to surgery and the underlying disease) consistent with the expected duration of ECMO support and management of DIC using new generation medications may help improve ECMO management.

\section{Conclusion}

Management of post-cardiotomy ECMO without heparinization rescued patients complicated with bleeding at a reasonable rate. However, serious DIC-related bleeding complications still occurred. Although we could only perform 
Table 2 Coagulation status

\begin{tabular}{|c|c|c|c|c|c|c|c|c|c|c|c|c|c|c|c|c|}
\hline \multirow[t]{2}{*}{ Patient \# } & \multicolumn{2}{|c|}{ DIC score } & \multicolumn{2}{|c|}{$\begin{array}{l}\text { D-Dimer }(\mu \mathrm{g} / \\
\mathrm{ml})\end{array}$} & \multicolumn{2}{|c|}{$\mathrm{FDP}(\mu \mathrm{g} / \mathrm{ml})$} & \multicolumn{2}{|c|}{ ATIII (\%) } & \multicolumn{2}{|c|}{$\begin{array}{l}\text { Fibrinogen } \\
(\mathrm{mg} / \mathrm{dl})\end{array}$} & \multicolumn{2}{|c|}{$\begin{array}{l}\text { Platelet }\left(\times 10^{4} /\right. \\
\mu \mathrm{l})\end{array}$} & \multicolumn{2}{|c|}{ PT-INR } & \multicolumn{2}{|l|}{ APTT } \\
\hline & Pre & Post & Pre & Post & Pre & Post & Pre & Post & Pre & Post & Pre & Post & Pre & Post & Pre & Post \\
\hline 1 & 2 & 5 & 0.8 & 33.3 & 0 & 87 & & 70 & 346 & 144 & 13.0 & 8.3 & 1.01 & 1.31 & 37.3 & 26.8 \\
\hline 3 & 1 & 6 & 1.0 & 28.8 & 4 & 83 & 88 & 67 & 238 & 382 & 9.5 & 14.9 & 1.26 & 1.31 & 43.4 & 29.9 \\
\hline 4 & 2 & 2 & 3.7 & 6.2 & 11 & 15 & 74 & 89 & 635 & 529 & 3.4 & 9.7 & 1.03 & 1.16 & 31.8 & 29.4 \\
\hline 6 & 2 & 3 & 4.6 & 8.3 & 11 & 78 & 85 & 94 & 371 & 229 & 9.3 & 7.9 & 1.23 & 1.13 & 37.0 & 37.8 \\
\hline 7 & 0 & 2 & 1.6 & 9.2 & & 21 & 56 & 54 & 393 & 254 & 15.6 & 13.1 & 1.38 & 1.32 & 52.5 & 39.9 \\
\hline 8 & 1 & 5 & 7.2 & 24.5 & 16 & 53 & 58 & 59 & 496 & 227 & 7.3 & 8.4 & 1.17 & 1.20 & 33.8 & 31.3 \\
\hline Mean & 1.3 & 3.8 & 3.2 & 18.4 & 8.4 & 56.2 & 72 & 72 & 413 & 294 & 9.7 & 10.4 & 1.18 & 1.24 & 39.3 & 32.5 \\
\hline SD & 0.8 & 1.7 & 2.5 & 11.9 & 6.3 & 31.9 & 15 & 16 & 137 & 138 & 4.3 & 2.9 & 0.14 & 0.09 & 7.6 & 5.2 \\
\hline$p$ value & 0.022 & & 0.033 & & 0.023 & & 0.951 & & 0.094 & & 0.714 & & 0.366 & & 0.042 & \\
\hline Median & 1.5 & 4.0 & 2.7 & 16.9 & 11.0 & 65.5 & 74 & 69 & 382 & 242 & 9.4 & 9.1 & 1.20 & 1.26 & 37.2 & 30.6 \\
\hline
\end{tabular}

$A P T T$ active partial thromboplastin time, ATIII antithrombin III, DIC disseminated intravascular coagulation, FDP fibrinogen degradation products, $P T$-INR prothrombin time international normalized ratio

post-cardiotomy ECMO without heparinization in such post-cardiotomy patients complicated with bleeding, more sophisticated management protocols are warranted to take into consideration risk of DIC, the presence of underlying disease, and expected duration of ECMO.

Acknowledgements The authors would like to thank Enago (http:// www.enago.jp) for the English language review.

\section{Compliance with ethical standards}

Conflict of interest There are no conflicts of interest to declare.

Open Access This article is distributed under the terms of the Creative Commons Attribution 4.0 International License (http://creativeco mmons.org/licenses/by/4.0/), which permits unrestricted use, distribution, and reproduction in any medium, provided you give appropriate credit to the original author(s) and the source, provide a link to the Creative Commons license, and indicate if changes were made.

\section{References}

1. Nakamura H, Yamaguchi H, Amano A, Nakao T. Venovenous extracorporeal membrane oxygenation is effective against postcardiotomy acute respiratory failure in adults. Gen Thorac Cardiovasc Surg. 2013;61:402-8.

2. Takagaki M, Yamaguchi H, Mitsuyama S, Kadowaki T, Ando T. Successful management of prolonged venovenous extracorporeal membrane oxygenation in an octogenarian. J Artif Organs. 2017;20:377-80.

3. Oliver WC. Anticoagulation and coagulation management for ECMO. Semin Cardiothorac Vasc Anesth. 2009;13(3):154-75.

4. Murphy DA, Hockings LE, Andrews RK, Aubron C, Gardiner EE, Pellegrino VA, et al. Extracorporeal Membrane Oxygenation Hemostatic Complications. Transfus Med Rev. 2015;29:90-101.

5. Fan E, Gattinoni L, Combes A, Schmidt M, Peek G, Brodie D,et al. Venovenous extracorporeal membrane oxygenation for acute respiratory failure. Intensive Care Med. 2016;42:712-24.
6. Nashef SAM, Roques F, Sharples LD, Nilsson J, Smith C, Goldstone AR, et al. EuroSCORE II. Eur J Cardiothorac Surg. 2012;41:734-45.

7. Nishida T, Masuda M. Risk prediction of cardiovascular surgery in Japanese patients. Gen Thorac Cardiovasc Surg. 2011;59:597-8.

8. Knaus WA, Draper EA, Wagner DP, Zimmerman JE. APACHE II: a severity of disease classification system. Crit Care Med. 1985;13(10):818-29.

9. Vincent JL, de Mendonça A, Cantraine F, Moreno R, Takala J, Suter PM, et al. Use of the SOFA score to assess the incidence of organ dysfunction/failure in intensive care units: results of a multicenter, prospective study. Working group on "sepsis-related problems" of the European Society of Intensive Care Medicine. Crit Care Med. 1998;26:1793-800.

10. Murray JF, Matthay MA, Luce JM, Flick MR. An expanded definition of the adult respiratory distress syndrome. Am Rev Respir Dis. 1988;138:720-3.

11. Schmidt M, Bailey M, Sheldrake J, Hodgson C, Aubron C, Rycus $\mathrm{PT}$, et al. Predicting survival after extracorporeal membrane oxygenation for severe acute respiratory failure: the respiratory extracorporeal membrane oxygenation survival prediction (RESP) score. Am J Respir Crit Care Med. 2014;189:1374-82.

12. Singh RK, Baronia AK, Sahoo JN, Sharma S, Naval R, Pandey $\mathrm{CM}$, et al. Prospective comparison of new Japanese Association for Acute Medicine (JAAM) DIC and International Society of Thrombosis and Hemostasis (ISTH) DIC score in critically ill septic patients. Thromb Res. 2012;129:e119-25.

13. Buscher H, Vukomanovic A, Benzimra M, Okada K, Nair P. Blood and anticoagulation management in extracorporeal membrane oxygenation for surgical and nonsurgical patients: a single-center retrospective review. J Cardiothorac Vasc Anesth. 2017;31:869-75.

14. Kreyer S, Muders T, Theuerkauf N, Spitzhüttl J, Schellhaas T, Schewe J, et al. Hemorrhage under veno-venous extracorporeal membrane oxygenation in acute respiratory distress syndrome patients: a retrospective data analysis. J Thorac Dis. 2017;9(12):5017-29.

15. Kasirajan V, Smedira NG, McCarthy JF, Casselman F, Boparai N, McCarthy PM. Risk factors for intracranial hemorrhage in adults on extracorporeal membrane oxygenation. Eur J Cardiothorac Surg. 1999;15:508-14. 